\title{
Article \\ Comparison of Camera Calibration and Measurement Accuracy Techniques for Phase Measuring Deflectometry
}

\author{
Renhao Ge ${ }^{1}$, Dahai $\mathrm{Li}^{1,2, *}$, Xinwei Zhang ${ }^{1}$, Ruiyang Wang ${ }^{1}$, Wanxing Zheng ${ }^{1}$, Xiaowei $\mathrm{Li}^{1}$ and Wuxiang Zhao ${ }^{1, *}$ \\ 1 School of Electronics and Information Engineering, Sichuan University, Chengdu 610065, China; \\ gerenhao@stu.scu.edu.cn (R.G.); xinwei_zhang@stu.scu.edu.cn (X.Z.); 2018322050019@stu.scu.edu.cn (R.W.); \\ 2019222050035@stu.scu.edu.cn (W.Z.); xwli@scu.edu.cn (X.L.) \\ 2 School of Aeronautics and Astronautics, Sichuan University, Chengdu 610065, China \\ * Correspondence: lidahai@scu.edu.cn (D.L.); zhaowuxiang@scu.edu.cn (W.Z.)
}

check for

updates

Citation: Ge, R.; Li, D.; Zhang, X.; Wang, R.; Zheng, W.; Li, X.; Zhao, W. Comparison of Camera Calibration and Measurement Accuracy Techniques for Phase Measuring Deflectometry. Appl. Sci. 2021, 11, 10300. https://doi.org/10.3390/ app112110300

Academic Editors: Cristian Focsa, Kijoon Lee and Giulio Nicola Cerullo

Received: 30 August 2021

Accepted: 30 October 2021

Published: 2 November 2021

Publisher's Note: MDPI stays neutral with regard to jurisdictional claims in published maps and institutional affiliations.

Copyright: (c) 2021 by the authors. Licensee MDPI, Basel, Switzerland. This article is an open access article distributed under the terms and conditions of the Creative Commons Attribution (CC BY) license (https:// creativecommons.org/licenses/by/ $4.0 /)$.

\begin{abstract}
Phase measuring deflectometry (PMD) is a competitive method for specular surface measurement that offers the advantages of a high dynamic range, non-contact process, and full field measurement; furthermore, it can also achieve high accuracy. Camera calibration is a crucial step for PMD. As a result, a method based on the calibration of the entrance pupil center is introduced in this paper. Then, our proposed approach is compared with the most popular photogrammetric method based on Zhang's technique (PM) and Huang's modal phase measuring deflectometry (MPMD). The calibration procedures of these three methods are described, and the measurement errors introduced by the perturbations of degrees of freedom in the PMD system are analyzed using a ray tracing technique. In the experiment, a planar window glass and an optical planar element are separately measured, and the measurement results of the use of the three methods are compared. The experimental results for the optical planar element (removing the first 6 terms of the Zernike polynomial) show that our method's measurement accuracy reached $13.71 \mathrm{~nm}$ RMS and $80.50 \mathrm{~nm} \mathrm{PV}$, which is comparable to accuracy values for the interferometer.
\end{abstract}

Keywords: phase measuring deflectometry; entrance pupil center; camera calibration; surface shape

\section{Introduction}

Phase measuring deflectometry (PMD) with structured light projection and the phaseshifting technique is a highly accurate optical surface measuring method based on the law of light reflection. It has the advantages of high speed, low cost, and a high dynamic range; furthermore, it requires a simple device and is non-destructive [1-10]. In the PMD system, rays are considered to be emitted from the projection center of a camera lens and reflected off a planar surface under test (SUT) to reach a liquid crystal display (LCD) screen. During the measurement process, the calibration step is carried out to establish the relationships among the screen coordinate system, the coordinate system of the SUT, and the camera coordinate system; then, the slope of the reflection points on the SUT are obtained according to the slope calculation equation $[9,10]$. Finally, the slope data are integrated to obtain the surface information [3-10]. Among these variables, the most important are the coordinate positions of the screen, the SUT and the projection center of the camera. The calibration error in them will degrade the measurement accuracy of the SUT.

At present, an external pinhole is utilized as the projection center of the camera, whose position can be accurately measured with a laser tracker in PMD or the Software Configurable Optical Test System (SCOTS) [3,11,12]. The projection center of the camera lens can also be calibrated based on the photogrammetry principle [13,14]. Based on Zhang's technique, Xiao et al. proposed a calibration method with a flat mirror to obtain the position of the projection center of the camera $[15,16]$. Additionally, Hou et al. proposed a method to calculate the position of the projection center in a unified world coordinate system from the external parameters (including the translation vector and rotation matrix) 
of the camera [16,17]. Zhang's technique requires taking several images from different orientations and different poses, but it is not always accessible in deflectometry, especially for a machine-integrated system.

Optically, the chief rays reflected off the SUT will certainly converge at the entrance pupil center of the camera lens [18-21]. The role of the projection center in the PMD system, as is mentioned in the references [13-17], is equivalent to this center. For the sake of distinction, we named it the entrance pupil center in our method, but it is still called the projection center in other methods.

For an actual lens, the position of the entrance pupil center can vary as the off-axis angle of an incoming ray increases [18]. However, for most of the lens, this effect is not significant, and thus it can usually be ignored in camera calibration [19]. Therefore, a method based on the calibration of the entrance pupil center is introduced in this paper. The entrance pupil calibration method (EPCM) that we propose is compared with the popular photogrammetric method based on Zhang's technique (PM) [16], and Huang's MPMD method (MPMD) [7]. A planar window glass about $90 \mathrm{~mm}$ in diameter and an optical planar element approximately $103 \mathrm{~mm}$ in diameter are measured separately by the three methods, and the measurement results are analyzed.

The rest of the paper is organized as follows: Section 2 illustrates the basic principle of the three methods (EPCM, PM, and MPMD). Section 3 describes the measurement procedures of the three methods. Section 4 presents the calculation of the perturbations of the degrees of freedom in the PMD system. Section 5 shows the experimental results, including the comparison of the camera calibration and measurement accuracy. Section 6 discusses and summarizes this paper.

\section{Principle}

\subsection{The Basic Principle of Phase Measuring Deflectometry}

The PMD system is composed of an LCD screen, a camera, and the SUT. Structured light patterns displayed by the screen are reflected by the SUT and captured by the camera. Then, the slope data can be calculated by the system's geometrical model and calibration parameters. Finally, the slope data is integrated to obtain the SUT shape. The coordinates of the screen pixels and the coordinates of the SUT instead of the reference surface are obtained for the triangulation calculation. The slope data of the SUT is calculated as shown in Equation (1) [10,22]:

$$
\begin{aligned}
& \tan \theta_{x}\left(x_{m}, y_{m}\right)=\frac{\frac{x_{m}-x_{s}}{d_{m 2 s}}+\frac{x_{m}-x_{c}}{d_{m 2 c}}}{\frac{z_{m 2 s}}{d_{m 2 s}}+\frac{z_{m 2 c}}{d_{m 2 c}}} \\
& \tan \theta_{y}\left(x_{m}, y_{m}\right)=\frac{\frac{y_{m}-y_{s}}{d_{m 2 s}}+\frac{y_{m}-y_{c}}{d_{m 2 c}}}{\frac{z_{m 2 s}}{d_{m 2 s}}+\frac{z_{m 2 c}}{d_{m 2 c}}}
\end{aligned}
$$

where $x_{m}$ and $y_{m}$ are the coordinates of the reflection points; $x_{s}$ and $y_{s}$ are the coordinates of the screen pixels, which can be calculated by the phase-shifting algorithm [23,24]; $x_{c}$ and $y_{c}$ are the coordinates of the camera; $z_{m 2 s}$ and $z_{m 2 c}$ are the distance between the SUT and the screen and distance between the SUT and the camera, respectively; and $d_{m 2 s}=$ $\sqrt{\left(x_{m}-x_{s}\right)^{2}+\left(y_{m}-y_{s}\right)^{2}+\left(z_{m 2 s}\right)^{2}}$ and $d_{m 2 c}=\sqrt{\left(x_{m}-x_{c}\right)^{2}+\left(y_{m}-y_{c}\right)^{2}+\left(z_{m 2 c}\right)^{2}}$. Then, the SUT shape can be obtained by integrating the slope data using the Zernike modal method [25-27].

\subsection{Methods}

\subsubsection{Entrance Pupil Calibration Method (EPCM)}

In Figure 1a, because both the entrance pupil center and the external stop lie on the optical axis in the paraxial region of the camera lens, a reference surface and an external stop are used to obtain the entrance pupil center, which is usually a virtual image of the aperture stop of the camera lens and is inside the lens. The origin of the global coordinate 
system $O-X Y Z$ is established as the point $O$, which is the coordinate of the external stop on the camera lens. Additionally, point $E$ is the entrance pupil center.

(a)

Reference surface

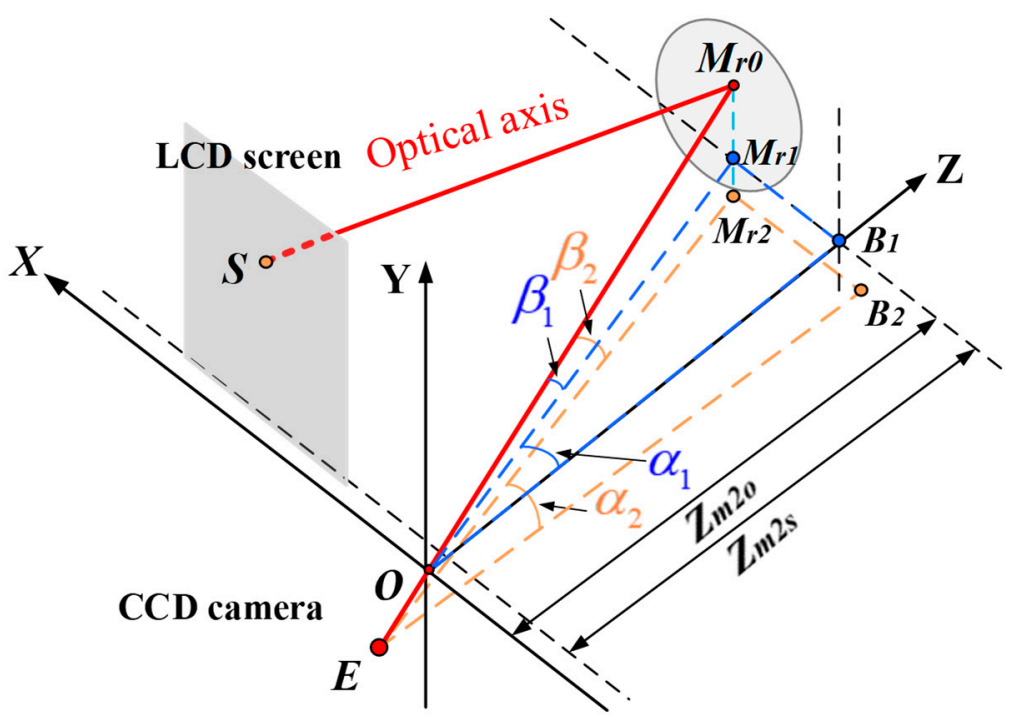

(b)

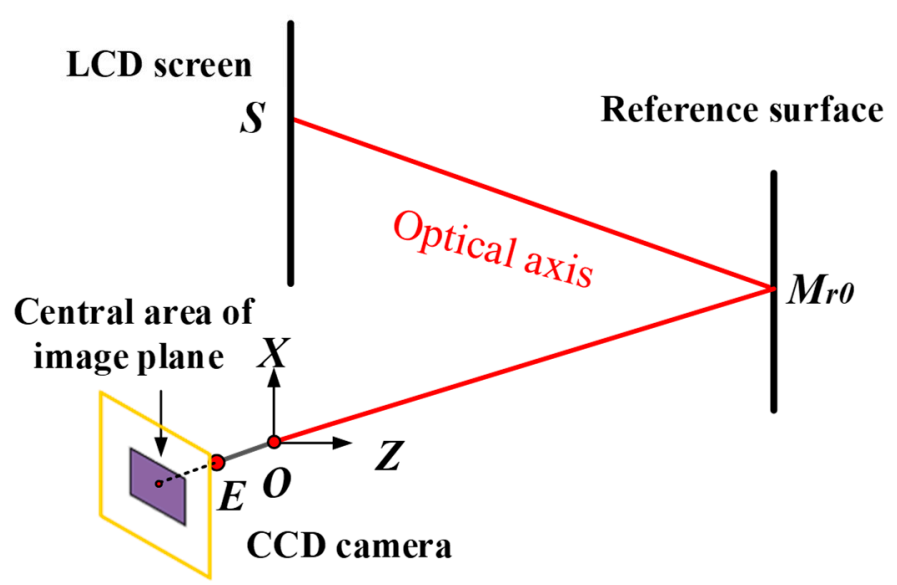

Figure 1. (a) Schematic diagram of the calibration of the entrance pupil center (point $O$ is the external stop, point $E$ is the entrance pupil center, and point $M_{r 0}$ is a reflection point on the reference surface); (b) illustration of central area of the image.

Based on the geometry shown in Figure 1a, where the points $E$ and $O$ are along with the optical axis, from the view of ray tracing in reverse we extend the ray of $E O$ to the reflection point $M_{r 0}\left(x_{m r 0}, y_{m r 0}\right)$ on the reference surface and reach spot $S$ on the LCD screen. These four points, $O, E, M_{r 0}$, and $S$, are along the optical axis. Assuming that the reference surface is an ideal plane, according to the law of reflection, the incident angle is equal to the reflection angle. The reflection points of the reference surface can be obtained, as shown in Equation (2):

$$
\begin{aligned}
& x_{m r}=\frac{x_{s r} z_{m 2 o}+x_{o} z_{m 2 s}}{z_{m 2 o}+z_{m 2 s}} \\
& y_{m r}=\frac{y_{s r} z_{m 2 o}+y_{o} z_{m 2 s}}{z_{m 2 o}+z_{m 2 s}}
\end{aligned},
$$

where $z_{m 2 s}$ and $z_{m 20}$ are the distance between the reference surface and the screen and the distance between the reference surface and point $O$ in the $z$ direction, respectively; $x_{s r}$ and $y_{s r}$ are the coordinates of the screen pixels corresponding to the central area of the image plane (as shown in Figure 1b); $x_{0}$ and $y_{0}$ are the coordinates of point $O$, which can 
be measured by the coordinate measuring machine (CMM). Significantly, point $M_{r 0}$ is one of the reflection points and is on the optical axis.

For convenience, the auxiliary points, $M_{r 1}, M_{r 2}, B_{1}$, and $B_{2}$, are depicted in Figure 1a. They are perpendicular to the $x$-axis and the $y$-axis, respectively. $M_{r 1}$ and $M_{r 2}$ are equal to point $O$ and point $E$ in the $Y$ direction, respectively. Similarly, $B_{1}$ and $B_{2}$ are equal to point $O$ and point $E$ in the $Y$ direction. As shown in Figure 1a, the angle relationships between the camera and the reference surface can be expressed in triangles $M_{r 1} O B_{1}$ and $M_{r 0} O M_{r 1}$, as shown in Equation (3):

$$
\begin{aligned}
& \alpha_{1}=\arctan \left(\frac{x_{m r 0}-x_{o}}{z_{m 2 o}}\right) \\
& \beta_{1}=\arctan \left(\frac{y_{m r 0}-y_{o}}{d_{m 2 o}}\right)
\end{aligned},
$$

where $d_{m 2 o}=\sqrt{\left(x_{m r 0}-x_{o}\right)^{2}+\left(y_{m r 0}-y_{o}\right)^{2}+\left(z_{m 20}\right)^{2}}$.

Based on triangles $M_{r 0} O M_{r 1}$ and $M_{r 0} E M_{r 2}$, triangles $M_{r 1} O B_{1}$ and $M_{r 2} E B_{2}$ are similar to each other. We assume that the distance between point $O$ and point $E$ is $d_{p}$. According to the above, the coordinates of the entrance pupil center can be determined in the global coordinate system, as shown in Equation (4):

$$
\begin{gathered}
d_{m 2 e}=d_{m 2 o}+d_{p} \\
z_{m 2 e}=d_{m 2 o} \cos \left(\beta_{2}\right) \cos \left(\alpha_{2}\right) \\
x_{e}=x_{m r 0}-z_{m 2 e} \tan \left(\alpha_{2}\right) \\
y_{e}=y_{m r 0}-d_{m 2 e} \sin \left(\beta_{2}\right) \\
z_{e}=z_{m 2 e}-z_{m 2 o}+z_{o}
\end{gathered}
$$

where $x_{e}, y_{e}$, and $z_{e}$ are the coordinates of the entrance pupil center, which are the coordinates of the camera, $\alpha_{2}=\alpha_{1}$ and $\beta_{2}=\beta_{1}$. After obtaining the camera coordinates, the reflection points coordinates can be solved using an iterative reconstruction strategy [28].

\subsubsection{Photogrammetric Method Based on Zhang's Technique (PM)}

In PM, the camera's internal and external parameters are calculated from feature points with known world coordinates and corresponding image coordinates. As a result, external parameters (rotation matrix $R$ and translation vector $T$ ) are obtained using calibration technology. Assuming that the world coordinates of the point $M=\left(X_{W}, Y_{W}, Z_{W}, 1\right)^{T}$ and its image coordinates $m=(u, v, 1)^{T}$ are known, the relationship between $\mathrm{M}$ and $\mathrm{m}$ can be described as

$$
s\left[\begin{array}{c}
u \\
v \\
1
\end{array}\right]=\left[\begin{array}{cccc}
f_{x} & \alpha & u_{0} & 0 \\
0 & f_{y} & v_{0} & 0 \\
0 & 0 & 0 & 0
\end{array}\right]\left[\begin{array}{cc}
R & T \\
0^{T} & 1
\end{array}\right]\left[\begin{array}{c}
X_{W} \\
Y_{W} \\
Z_{W} \\
1
\end{array}\right]=A\left[\begin{array}{cc}
R & T \\
0^{T} & 1
\end{array}\right]\left[\begin{array}{c}
X_{W} \\
Y_{W} \\
Z_{W} \\
1
\end{array}\right],
$$

where $f_{x}$ and $f_{y}$ are the equivalent focal lengths in pixels along the $x$ and $y$ directions, $\alpha$ is a skew factor, and $\left(u_{0}, v_{0}\right)$ are the coordinates of the principal point. These parameters are called the camera intrinsic parameters $A$. The rotation matrix $R$ and translation vector $T$ are the external parameters. In Hou's method [17], assuming the coordinates of a point are $M_{c}$ in the camera coordinate system and $M_{w}$ in the world coordinate system, the relationship between $M_{\mathcal{c}}$ and $M_{w}$ is expressed by the following equation:

$$
M_{c}=R M_{W}+T,
$$

The coordinates of the projection center in the camera coordinate system are $(0,0,1)$, and its world coordinates can be obtained by the following equation,

$$
M_{W}=R^{-1}\left(M_{c}-T\right) .
$$


In E's method [10], which can achieve a high measurement accuracy, a checkboard is displayed on another LCD screen with several different orientations, the camera takes pictures of the checkboard in different poses, and matrix $A$ and $[R T]$ are obtained with the calibration toolbox [29]. Hence, Equation (8) can be obtained by substituting $M=$ $\left(X_{W}, Y_{W}, Z_{W}, 1\right)^{T}$ in Equation (5) with $M=\left[x_{m}, y_{m}, 0,1\right]^{T}$ :

$$
s\left[\begin{array}{l}
u \\
v \\
1
\end{array}\right]=A\left[\begin{array}{cc}
R & T \\
0^{T} & 1
\end{array}\right]\left[\begin{array}{c}
x_{m} \\
y_{m} \\
0 \\
1
\end{array}\right]=A\left[\begin{array}{lll}
r_{1} & r_{2} & t
\end{array}\right]\left[\begin{array}{c}
x_{m} \\
y_{m} \\
1
\end{array}\right]=H\left[\begin{array}{c}
x_{m} \\
y_{m} \\
1
\end{array}\right] .
$$

It is worth noting that the last pose of the checkerboard plane is located at the identical position as the SUT for getting rid of misalignment, as it makes the local coordinate system on the last checkerboard parallel to each axis of the global coordinate system. Since $H$ is a homography matrix in Equation (8), the mirror pixel coordinates can be determined using the following equation:

$$
\left[\begin{array}{c}
x_{m} \\
y_{m} \\
1
\end{array}\right]=s H^{-1}\left[\begin{array}{l}
u \\
v \\
1
\end{array}\right]
$$

In addition, the system errors can be eliminating by measuring a high-quality reference surface that has a known shape and is aligned at an identical position to the SUT [10]. This measurement can be mathematically stated as follows:

$$
P_{t}-P_{r}+I_{r}=\left(I_{t}+\text { error_t } t\right)-\left(I_{r}-\text { error_t } t\right)+I_{r} \approx I_{t},
$$

where $P_{t}$ and $P_{r}$ are the measurement of the SUT and the reference surface, respectively, and are directly obtained from the PMD measurement method, which contains the system errors error $\_$t and error $\_r$, respectively. $I_{t}$ is the measured surface shape of the test flat after the system errors are eliminated. $I_{r}$ is the surface shape of the reference element and can be measured by Fizeau interferometer.

\subsubsection{Modal Phase Measuring Deflectometry (MPMD)}

In MPMD, the height and slopes of the SUT are represented by the well-established mathematical model and updated by optimizing the model coefficient $\mathbf{c}$ to minimize the discrepancy between the re-projection in ray tracing and the actual measurement [7]. As shown in Figure 2, the incident ray $\vec{p}$ is reflected off the SUT to the screen, and the reflected ray $\vec{r}$ can be obtained with Equation (11):

$$
\vec{r}=\vec{p}-2\langle\vec{p}, \vec{n}\rangle \vec{n}
$$

where $\vec{n}, \vec{p}$, and $\vec{r}$ are the unit vector of the normal, the incident ray and the reflected ray, respectively, and $<>$ represents the dot product.

The position of the screen relative to the camera (rotation matrix $R_{m}$, translation vector $T_{m}$ ) can be obtained through a pre-calibration procedure, which includes camera calibration, screen calibration, and geometric calibration. Once the pose of the screen is initialized via pre-calibration, the corresponding intersection $S^{\tau}$ of the screen is calculated. The coefficient vector $\mathrm{c}$ is initialized using the mathematical model of the SUT.

The coefficient vector $\mathbf{c}$ and the pose of the screen $\left(R_{m}, T_{m}\right)$ are optimized by minimizing the differences between the re-projection $S^{\tau}$ and the measurement $S$ on the screen among the $N$ pairs of correspondence in the least squares sense. The nonlinear least squares problem in Equation (12) can be solved using the Levenberg-Marquardt algorithm.

$$
\left[R_{n}^{\tau}, T_{n}^{\tau}, c^{\tau}\right]=\arg \min _{R_{m}, T_{m}, c} \sum_{n=1}^{N}\left\|S_{n}^{\tau}\left(R_{m}, T_{m}, c\right)-S_{n}\right\| .
$$




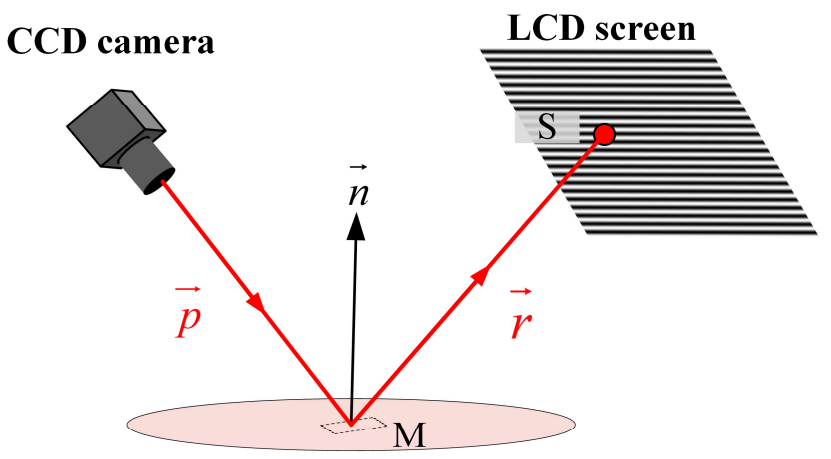

Specular surface

Figure 2. Sketches of MPMD can be drawn in ray tracing with a probe from the camera.

\section{Measurement Procedures of Various Methods}

\subsection{EPCM}

According to the principle of the entrance pupil calibration, the position of an external stop on the camera is measured. The EPCM is introduced in detail as follows:

(a) The global coordinate system $O-X Y Z$ is established with $O$ point as the origin. Then, point $O$ and distance $d_{p}$ between the camera and the external stop in the $\mathrm{z}$ direction are determined;

(b) The fringe pattern on the screen is reflected off the reference surface and captured by a camera equipped with an external stop. The screen coordinates corresponding to the central area of the image plane are calculated using the phase-shifting algorithm. The central area of the image plane with $2 \mathrm{M} \times 2 \mathrm{M}$ pixels ( $\mathrm{M}$ is a positive integer) is shown in Figure 1b. We calculate and obtain the coordinates of the reflection points of the reference surface using Equation (2); the coordinates of point $M_{r 0}$ can be obtained by the average of the reflection points corresponding to the central area of the image;

(c) The distance $d_{p}$ between point $O$ and point $E$ can be measured in the experiment. The coordinates of point $E$ can be obtained by Equations (3) and (4) in the global coordinates;

(d) The points of the reference surface are used as the initial values of the reflection points;

(e) The slope is calculated using Equation (1) and the reconstruction height $\mathrm{w}$ from the slope. It is output after the height is less than the threshold;

(f) The surface shape is reconstructed.

The calibration procedure of EPCM is illustrated in Figure 3.

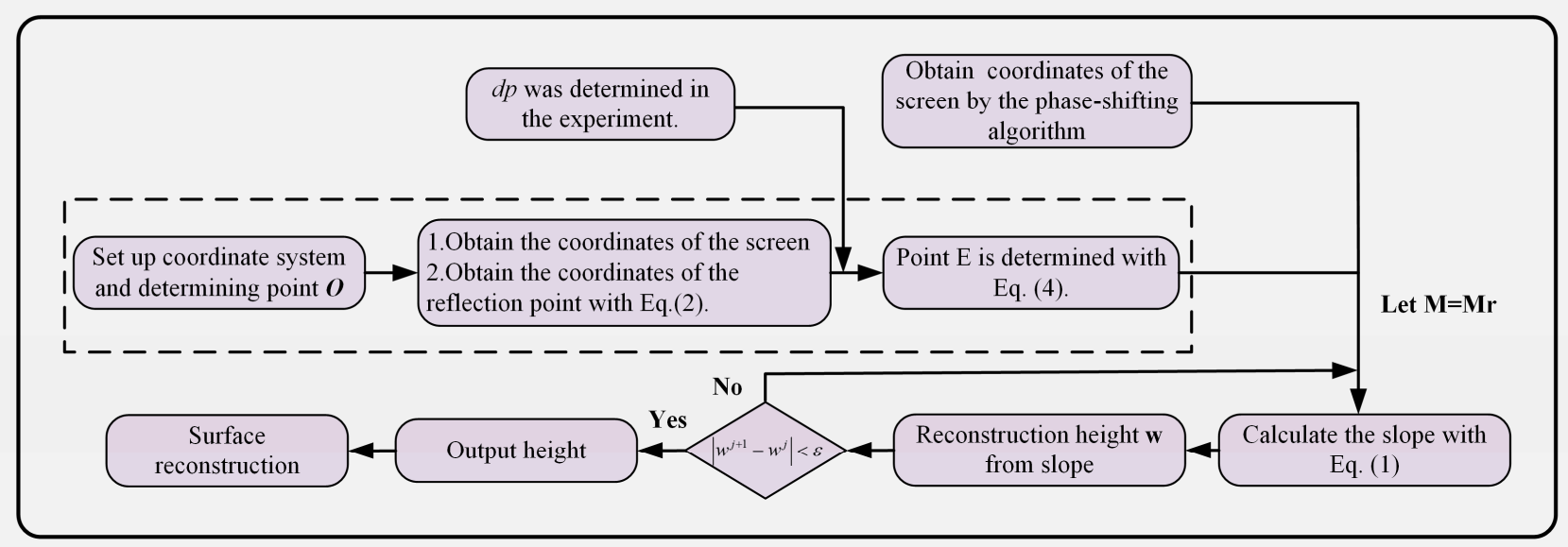

Figure 3. Flow chart of EPCM. 


\section{2. $P M$}

The procedure of PM is introduced in detail as follows:

(a) The test system is established. It is worth noting that the last pose of the checkerboard plane is located at an identical position to the SUT, and it makes the local coordinate system on the last checkerboard parallel to each axis of the global coordinate system;

(b) The rotation matrix $R$ and translation vector $T$ are obtained by camera calibration;

(c) The projection center is obtained with Equation (7), and the coordinates of the SUT are calculated with Equation (9);

(d) The slope is calculated with Equation (1), and the surface shape of the reference surface and the SUT are reconstructed;

(e) The systematic error is eliminated with Equation (10). The procedure of PM is illustrated in Figure 4a.

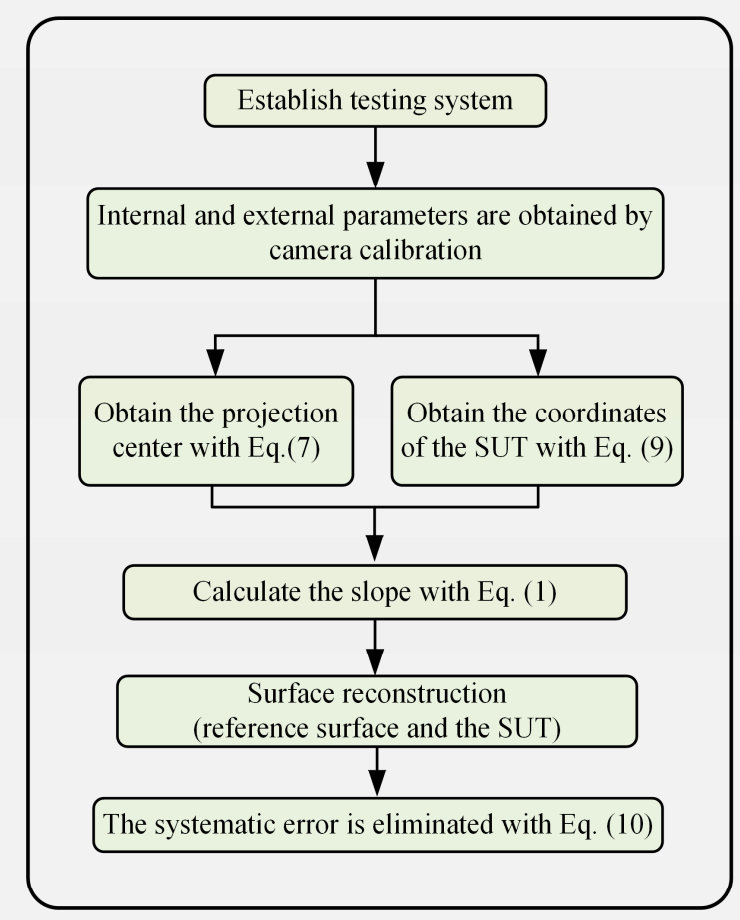

(a)

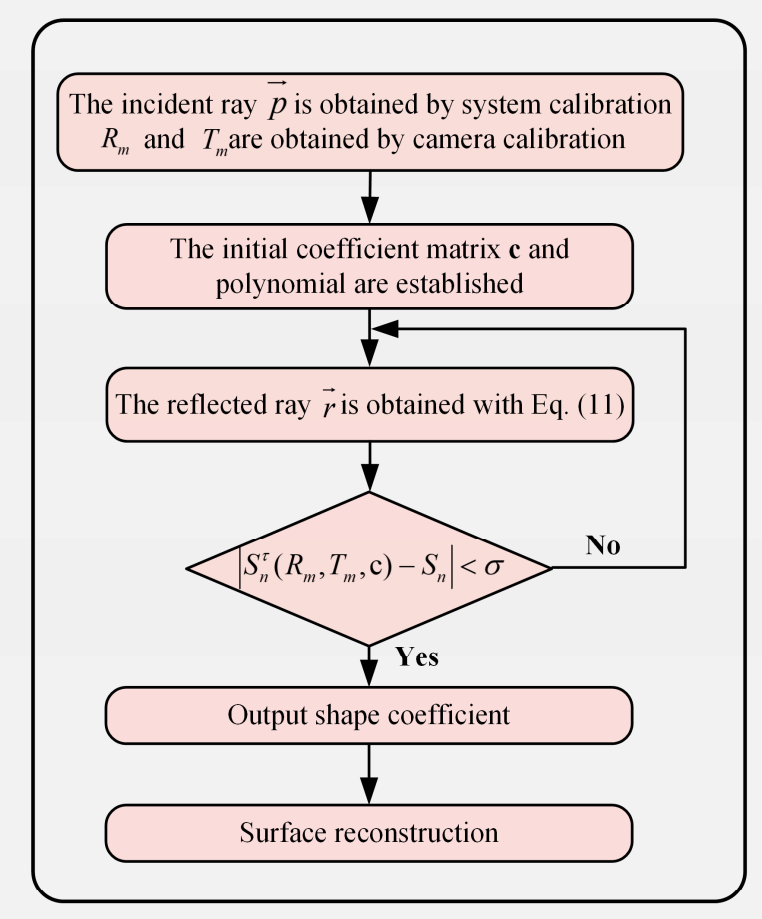

(b)

Figure 4. Flow charts: (a) PM; (b) MPMD.

\subsection{MPMD}

The procedure of MPMD is introduced in detail as follows:

(a) The incident ray $\vec{p}$ is calibrated using the system calibration. The pose of the screen relative to the camera $\left(R_{m}, T_{m}\right)$ can be obtained through the pre-calibration procedure;

(b) The initial coefficient matrix $\mathbf{c}$ and Zernike polynomial are used to represent the surface shape of the SUT;

(c) The incident ray $\vec{p}$ is reflected from the SUT to the screen. The reflected ray $\vec{r}$ can be calculated with Equation (5), and the intersection $S^{\tau}$ on the screen is obtained;

(d) The coefficient vector $\mathbf{c}$ and pose of the screen $\left(R_{m}, T_{m}\right)$ are optimized by minimizing the differences between the re-projection $S^{\tau}$ and the measurement $S$ of the screen among the $\mathrm{N}$ pairs of correspondence in the least squares sense;

(e) Output the shape coefficient and reconstruct the surface shape.

The procedure of MPMD is illustrated in Figure $4 \mathrm{~b}$. 


\section{Accuracy Analysis}

The following section describes the analysis of the measurement errors due to the perturbations of the degrees of freedom in the PMD system. Generally, the measurement result depends on the accuracy of the measurement tool and device. Therefore, it is necessary to analyze measurement errors caused by each coordinate value, which is called the degree of freedom (DOF). The error analysis aids in the identification of which DOF need to be tightened and which might be loosened. Thus, there are three DOF $\left(x_{c}, y_{c}, z_{c}\right)$ for the camera coordinates. For the coordinate measurement of the screen pixels, there are six DOFs; these are translations $\left(x_{s}, y_{s}, z_{s}\right)$ and rotations (screen tilt $x$, screen tilt $y$, screen tilt $z$ ) in three directions. For the coordinate measurement of the SUT, the rotation of the SUT around the $z$ axis does not cause measurement error; only the measurement results need to be rotated. Therefore, there are five DOFs with three translations $\left(x_{m}, y_{m}, z_{m}\right)$ and two rotations (flat tilt $x$, flat tilt $y$ ) for the SUT. Regardless of the errors by the LCD screen, such as thermal distortion, roughness, and noise, the 14 DOFs need to be considered [9].

As mentioned above, the DOFs are analyzed in the simulation. The schematic diagram of the PMD system is shown in Figure 5. Assuming that the pinhole $O$ coordinates are the origin of the world coordinate system, the distance between the SUT and the camera $O$ is $1525 \mathrm{~mm}$, and the distance between the LCD screen and the SUT is $1500 \mathrm{~mm}$ in the $z$ direction, while the angle between the camera and the $z$ direction is $5^{\circ}$. Korsch ray tracing [30] is used to calculate the slope of an ideal planar element in the $\mathrm{x}$ and $\mathrm{y}$ directions. For DOF, initial values are considered to be the accurate values. For the coordinate values of the camera $\left(x_{c}, y_{c}, z_{c}\right)$, the screen pixel $\left(x_{s}, y_{s}, z_{s}\right)$, and the SUT $\left(x_{m}, y_{m}, z_{m}\right)$, a perturbation of $1 \mathrm{~mm}$ is artificially added to the accurate value of each DOF, respectively. Similarly, a perturbation of $1 \mathrm{mrad}$ is artificially added to the accurate value for the five DOFs of the rotations, respectively. When error is added artificially to each DOF, the others remain unchanged.

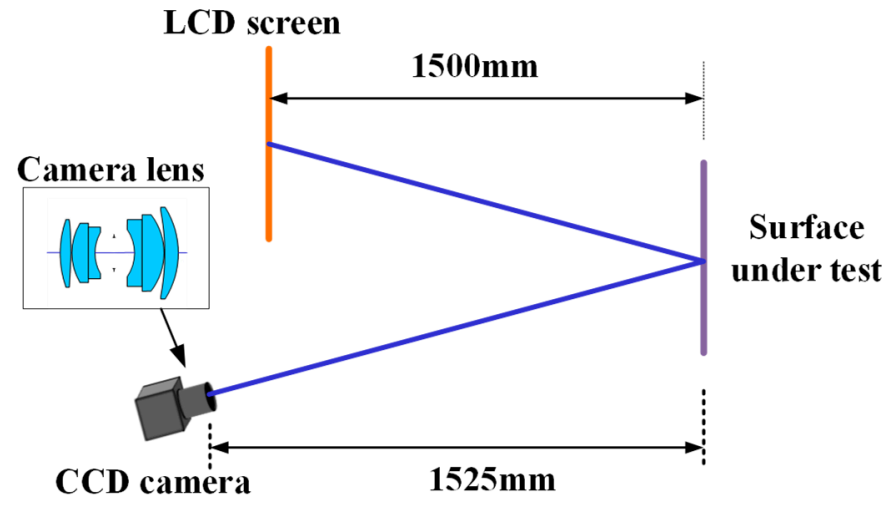

Figure 5. Schematic diagram of PMD.

Based on the above analysis, the Zernike polynomial coefficients are analyzed after the surface shape is reconstructed with the Zernike polynomial $[25,26]$ under different DOFs with errors. The differences from the 4 th to 11th terms of the Zernike polynomial coefficient between the results of the accurate surface figure and the results gained using different perturbations are shown in Table 1. The terms from the 4th to 11th Zernike polynomial coefficients are defocus, oblique astigmatism, vertical astigmatism, vertical coma, horizontal coma, vertical trefoil, oblique trefoil, and primary spherical, respectively. The root sum square (RSS) is the value for evaluating the effects on the measurement results due to the perturbation of each DOF or in every term of the Zernike polynomial coefficient. 
Table 1. The difference in Zernike coefficient caused by the disturbance of each degree of freedom. Unit: $\mathrm{nm}$.

\begin{tabular}{ccccccccccc}
\hline Zernike Terms/Degree of Freedom & Z4 & Z5 & Z6 & Z7 & Z8 & Z9 & Z10 & Z11 & RSS \\
\hline & $x_{c}$ & 20.92 & -0.01 & 9.75 & 0.00 & 2.27 & 0.00 & -0.03 & -0.01 & 23.19 \\
& $y_{c}$ & -0.11 & 9.91 & 0.05 & 2.32 & 0.00 & -0.03 & 0.00 & 0.00 & 10.18 \\
\multirow{2}{*}{$\begin{array}{c}\text { The error of 1 mm is } \\
\text { artificially }\end{array}$} & $z_{c}$ & 160.06 & 0.00 & -1.73 & 0.00 & -0.54 & -1.66 & 0.00 & -0.04 & 160.08 \\
introduced, & $x_{m}$ & -42.34 & 0.03 & -19.74 & 0.00 & -4.58 & 0.00 & 0.06 & 0.02 & 46.93 \\
respectively & $y_{m}$ & 0.06 & -19.98 & -0.03 & -4.68 & 0.00 & 0.05 & 0.00 & 0.00 & 20.52 \\
& $z_{m}$ & -2.67 & 0.00 & 0.03 & 0.00 & 0.00 & 0.00 & 0.00 & 0.00 & 2.67 \\
& $x_{s}$ & 21.43 & -0.01 & 9.99 & 0.00 & 2.30 & 0.00 & -0.03 & -0.01 & 23.76 \\
& $y_{s}$ & 0.07 & 10.24 & -0.04 & 2.40 & 0.00 & -0.03 & 0.00 & 0.00 & 10.52 \\
& $z_{s}$ & -162.73 & 0.00 & 1.75 & 0.00 & 0.55 & 0.00 & 0.00 & 0.05 & 162.74 \\
\hline \multirow{2}{*}{$\begin{array}{c}\text { The error of 1 mrad } \\
\text { is artificially }\end{array}$} & Screen tilt $x$ & -0.12 & 0.00 & 0.18 & 0.00 & 0.00 & 0.00 & 0.00 & 0.00 & 0.22 \\
introduced, & Screen tilt $y$ & -0.12 & 0.00 & -0.17 & 0.00 & 0.00 & 0.00 & 0.00 & 0.00 & 0.21 \\
respectively & Screen tilt $z$ & -0.10 & 352.02 & -0.17 & -0.52 & 0.00 & -0.42 & 0.00 & 0.00 & 352.02 \\
& flat tilt $x$ & 0.12 & 0.00 & -0.18 & 0.00 & 0.00 & 0.00 & 0.00 & 0.00 & 0.22 \\
& flat tilt $y$ & 0.12 & 0.00 & 0.17 & 0.00 & 0.00 & 0.00 & 0.00 & 0.00 & 0.21 \\
\hline
\end{tabular}

In general, the first eight terms of the Zernike polynomial coefficients are greatly affected when the DOF can be not obtained accurately. Therefore, according to the pre-set perturbation for all DOFs shown in the first column in Table 1, the accuracy of the alignment can be estimated in this measuring system. When the positions of the camera, the screen, and the SUT are separately changed under a given perturbation, these alignment errors for the 4th to the 11th Zernike polynomial coefficients can be calculated, as shown in Table 1. This table shows that the coordinate error of $z_{e}$ and $z_{s}$ will induce some relatively large errors in terms of defocus, while screen tilt error about the $\mathrm{z}$ axis will produce relatively significant errors in terms of astigmatism; however, the other terms are so small that they can be ignored.

\section{Experiment Results}

\subsection{Comparison of Camera Calibration}

The coordinates of the screen are obtained by the phase-shifting algorithm in three methods. For EPCM, the distance $d_{p}$ between the external stop and the front surface is obtained as $d_{p}=23.96 \mathrm{~mm}$ (see Appendix A). The experimental setup of the PMD is shown in Figure 6, where the LCD screen on the optical experiment platform displays a sixteenstep sinusoidal fringe pattern. The resolution of the CCD camera is $1296 \times 966$ pixels, and one pixel is $3.75 \mu \mathrm{m}$. The light emitted from the LCD screen is reflected by a high-quality flat element and captured by a camera with an external stop, which can be removed. The coordinates of the external stop can be accurately positioned with CMM and the camera focus on the element regardless of the screen being out of focus.

Three methods, including EPCM, PM, and MPMD, are selected for comparison. In EPCM, the camera coordinates are obtained with Equation (4) according to the geometric system in Figure 1a. In PM, the 16 poses of the checkerboard are captured (the last one is restored at an identical position to the SUT); then, the internal and external parameters are obtained by the calibration toolbox [29]. Finally, the projection center of the camera is calculated with Equation (7). Likewise, in MPMD, Xiao's algorithm [15] is used to obtain the relationship between the camera and the screen. Additionally, we then calculate the camera coordinates with Equation (7), which is equivalent to the PM in principle. According to the calibration processes mentioned above, the camera coordinates are obtained in EPCM, MPMD, and PM, respectively. Additionally, they are transformed into the same coordinate system, as shown in Table 2. It can be seen from Table 2 that the camera coordinates obtained by using the above three methods are different in the $x, y$, and $z$ directions. According to Table 1 , if the camera coordinate in the $\mathrm{x}$ direction is inaccurate, the error will mainly be induced in the 4th and 6th terms of the Zernike polynomial; inaccuracy in the 
$y$ direction will be largely embodied in the 5th term; and error in the $\mathrm{z}$ direction will be expressed in the 4th term and will have a great influence on the reconstruction accuracy. Therefore, if the three sets of coordinates in Table 1 are separately used to measure the SUT, the inaccuracy of the camera coordinates will be mostly reflected in the defocus and astigmatism error.

Table 2. Camera coordinates are obtained in three methods (EPCM, PM and MPMD). Unit: mm.

\begin{tabular}{cccc}
\hline Methods/Coordinates & $x_{c}$ & $y_{c}$ & $z_{c}$ \\
\hline EPCM & 344.073 & 162.974 & -17.021 \\
PM & 338.571 & 162.928 & -21.154 \\
MPMD & 339.900 & 162.334 & -17.994 \\
\hline
\end{tabular}

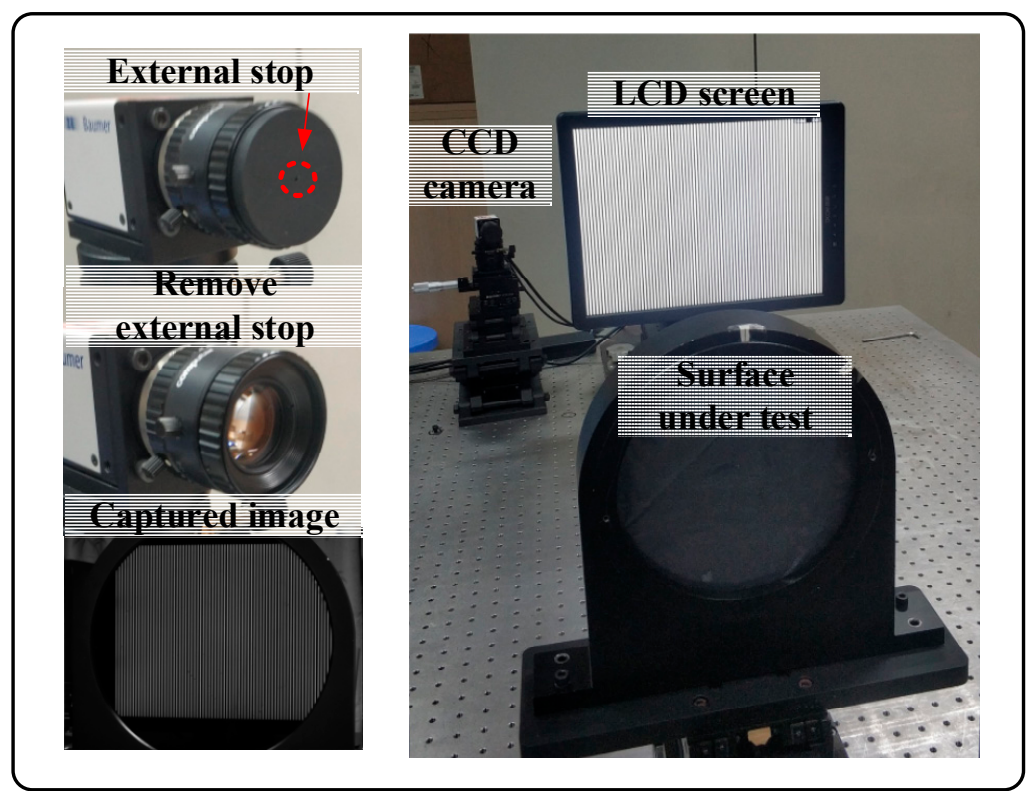

Figure 6. Experimental setup of PMD based on the entrance pupil center.

\subsection{Comparison of Measurement Accuracy}

In the experiments, the three methods (EPCM, PM, and MPMD) are used to measure the planar window glass with a diameter of about $90 \mathrm{~mm}$. According to the above three methods, the surface shape results of the planar window glass are obtained after removing the first three terms of Zernike polynomials, as shown in Figure 7. Figure 7a-e shows the reconstruction results obtained by the EPCM, PM with systematic errors, the PM after eliminating systematic errors, and the MPMD. The measurement result obtained by the interferometer are shown in Figure 7e, and its root-mean-square (RMS) and peak-to-valley (PV) values are $519.77 \mathrm{~nm}$ and $2328.59 \mathrm{~nm}$. 


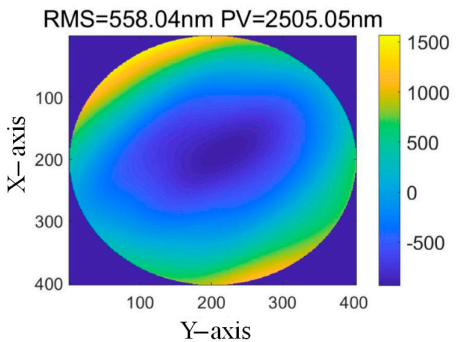

(a)

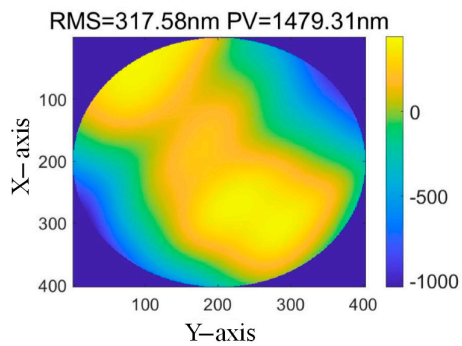

(b)

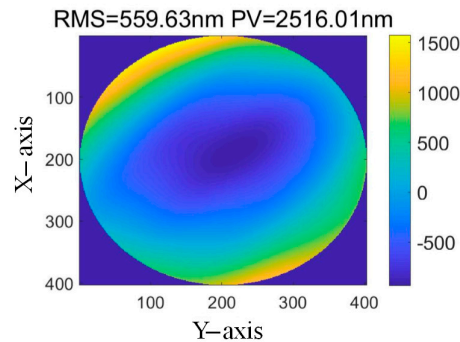

(c)

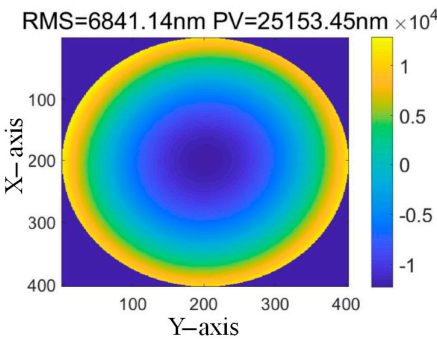

(d)

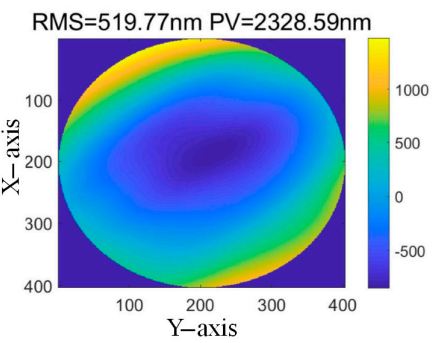

(e)

Figure 7. Experiment results of the planar window glass (after removing the first three terms): (a) EPCM; (b) PM with systematic errors; (c) PM after eliminating systematic errors; (d) MPMD; (e) Interferometer.

The surface shape shown in Figure 7a is closest to the interferometer, and its RMS value and PV value are $558.04 \mathrm{~nm}$ and $2505.05 \mathrm{~nm}$, respectively. Compared with the interferometer, the RMS and PV values of the surface shape in Figure $7 \mathrm{~b}$ are $317.58 \mathrm{~nm}$ and $1479.31 \mathrm{~nm}$, and the inaccuracy of the defocus term is directly presented. However, the surface shape of Figure $7 \mathrm{c}$ is obtained by eliminating the systematic error of Figure $7 \mathrm{~b}$, and its RMS value and PV value are $559.63 \mathrm{~nm}$ and $2516.01 \mathrm{~nm}$, respectively. This is closer to the interferometer than that in Figure $7 \mathrm{~b}$. In Figure $7 \mathrm{~d}$, compared with interference, it can be seen that there are serious errors in both defocus and astigmatism.

According to the analysis in Table 1, not only the inaccuracy of the camera coordinates but also the rotation of the screen around the $z$-axis has a great impact on the reconstruction results, which introduce errors in astigmatism. As a result, the reconstruction results of the planar window glass are obtained after removing the first 6 terms of Zernike polynomials, as shown in Figure 8. I It can be seen from Figure 8 that Figure $8 \mathrm{a}-\mathrm{c}$ are relatively closer to Figure 8e, while the low older error in Figure $8 \mathrm{~d}$ is not yet removed due to the initial value choice of the shape coefficient and accuracy limit of the method. In a word, for the PM with systematic errors and MPMD, error of the low-order term is generated due to inaccurate coordinates, but the EPCM still has a high measurement accuracy.

To further compare their accuracy performance, an optical planar element with a diameter of about $103 \mathrm{~mm}$ is measured. The surface shapes are obtained after removing the first three terms of the Zernike polynomials, as shown in Figure 9. Compared with the interferometer, the errors in Figure 9a,c are mainly reflected in the astigmatism term. It can be seen from Table 1 that the error is mainly caused by the rotation of the screen around the $z$-axis. Both Figure $9 b$,d have serious errors in terms of the defocus. These are mainly caused by the inaccuracy of $z_{c}$ and $z_{s}$, according to Table 1 . In order to remove the errors mentioned above, the surface shapes are obtained after removing the first six terms of the Zernike polynomials, as shown in Figure 10. It can be directly shown from Figure 10 that Figure 10a,c are comparable to the interferometers in shapes and magnitudes. Due to the limitation of calibration accuracy, both Figure 10b, d have significant errors compared with the interferometer. Additionally, in our previous work (see Appendix A), for the same element, the results of removing the first four terms were obtained, and the RMS and PV were $27.70 \mathrm{~nm}$ and $187.40 \mathrm{~nm}(f / 8)$, respectively, which are very close to the interferometer results (24.86 nm RMS and $154.73 \mathrm{~nm}$ PV). In order to compare the accuracy of the three 
methods more clearly in these two experiments, the measurement results are summarized in Table 3.

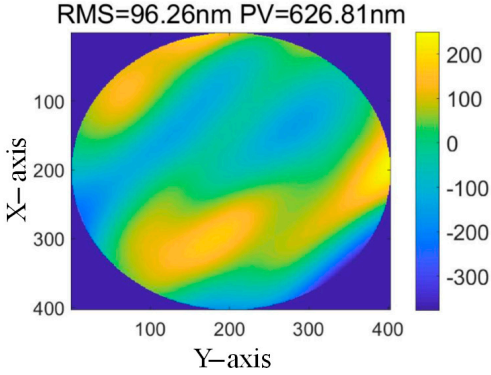

(a)

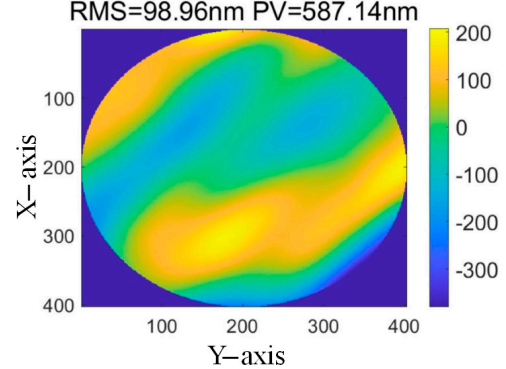

(b)

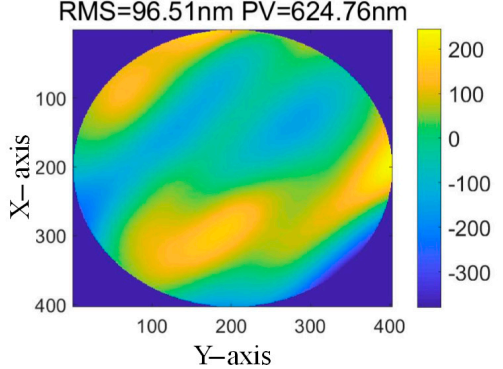

(c)

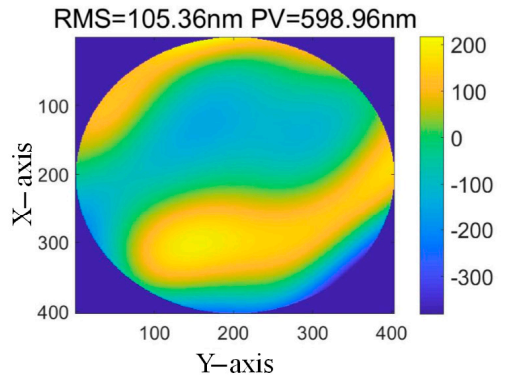

(d)

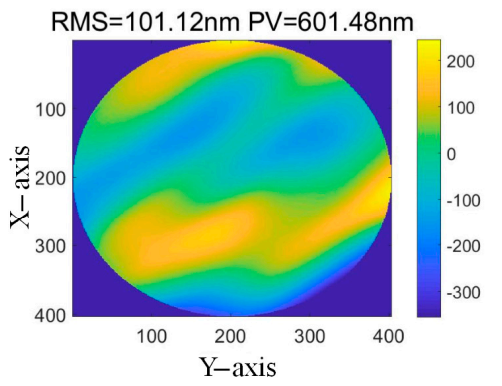

(e)

Figure 8. Experiment results of the planar window glass (after removing the first six terms): (a) EPCM; (b) PM with systematic errors; (c) PM after eliminating systematic errors; (d) MPMD; (e) Interferometer.
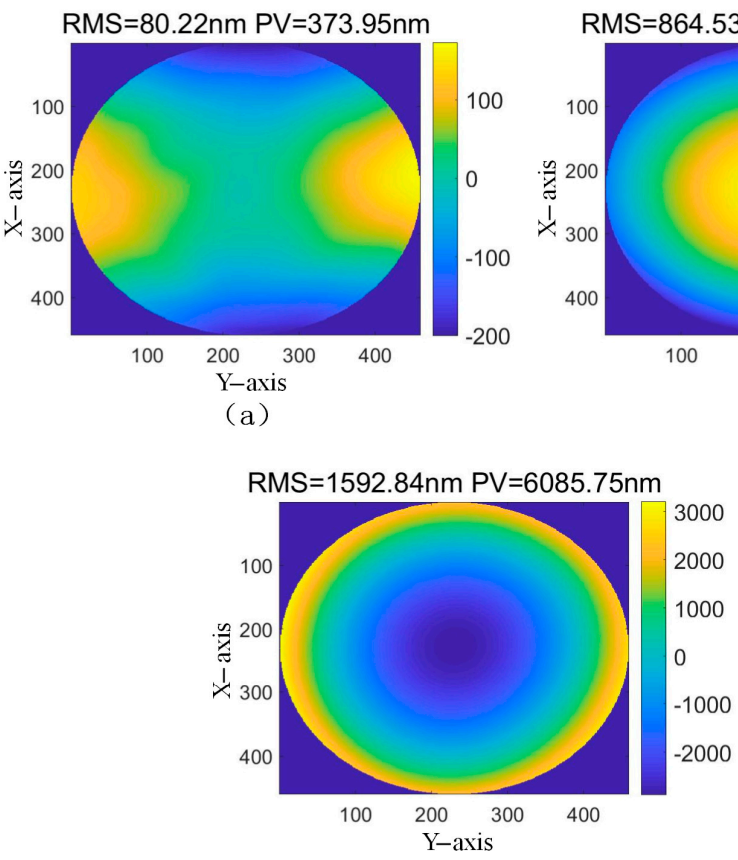

(d)

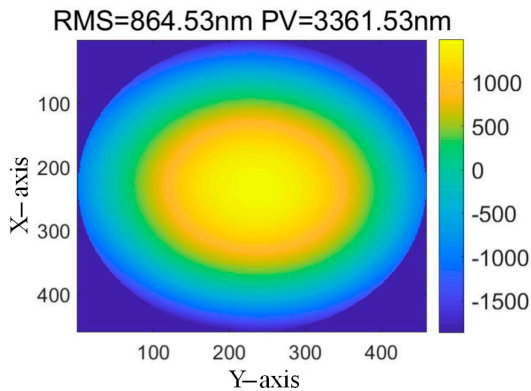

(b)
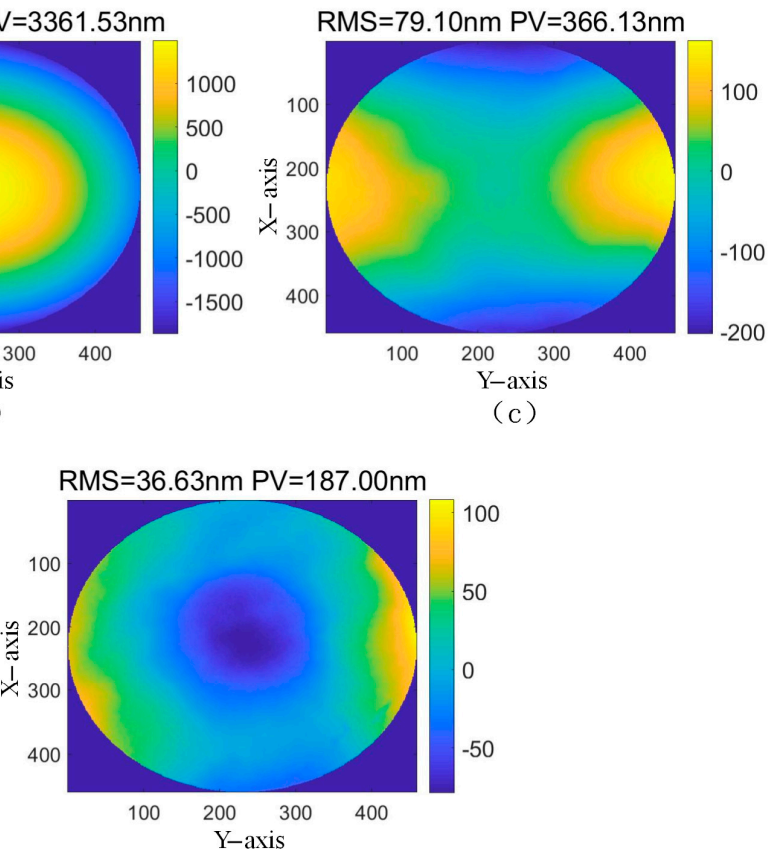

(e)

Figure 9. Experiment results of the optical planar element (after removing the first three terms): (a) EPCM; (b) PM with systematic errors; (c) PM after eliminating systematic errors; (d) MPMD; (e) Interferometer. 


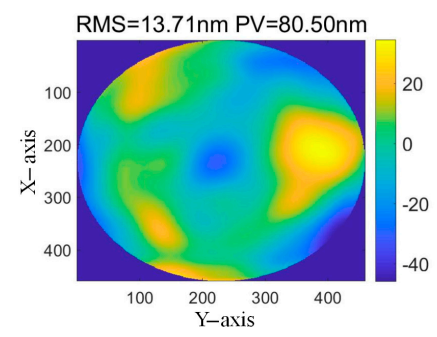

(a)

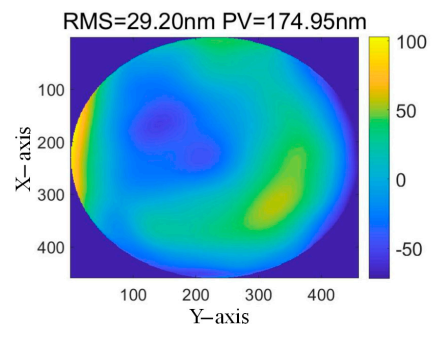

(b)

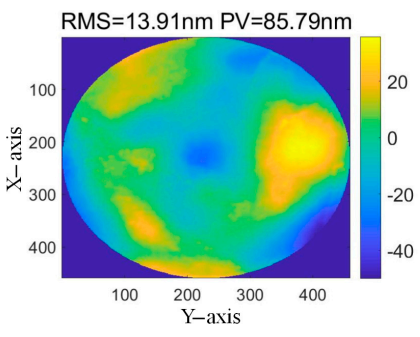

(c)

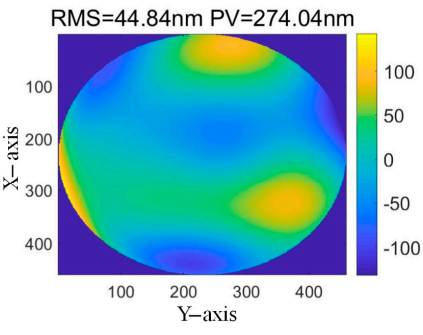

(d)

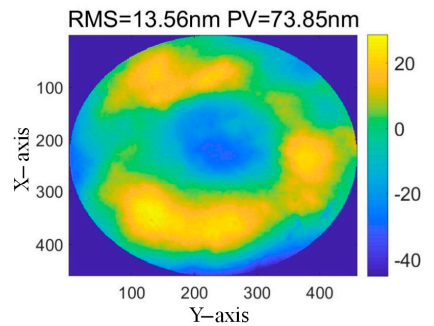

(e)

Figure 10. Experiment results of the optical planar element (after removing the first six terms): (a) EPCM; (b) PM with systematic errors; (c) PM after eliminating systematic errors; (d) MPMD; (e) Interferometer.

Table 3. Experiment results of the planar window glass and the optical planar element. Unit: nm.

\begin{tabular}{|c|c|c|c|c|c|c|c|c|c|c|c|}
\hline \multirow{2}{*}{\multicolumn{2}{|c|}{ Experiments/Methods }} & \multicolumn{2}{|c|}{ EPCM } & \multicolumn{2}{|c|}{$\begin{array}{c}\text { PM with } \\
\text { Systematic Errors }\end{array}$} & \multicolumn{2}{|c|}{$\begin{array}{c}\text { PM after } \\
\text { Eliminating } \\
\text { Systematic Errors }\end{array}$} & \multicolumn{2}{|c|}{ MPMD } & \multicolumn{2}{|c|}{ Interferometer } \\
\hline & & RMS & PV & RMS & PV & RMS & PV & RMS & PV & RMS & PV \\
\hline \multirow[t]{2}{*}{1} & $\begin{array}{c}\text { Removing the first } \\
3 \text { terms }\end{array}$ & 558.04 & 2505.05 & 317.58 & 1479.31 & 559.63 & 2516.01 & 6841.14 & $15,153.45$ & 519.77 & 2328.59 \\
\hline & $\begin{array}{l}\text { Removing the first } \\
6 \text { terms }\end{array}$ & 96.26 & 626.81 & 98.96 & 587.14 & 96.51 & 624.76 & 105.36 & 598.96 & 101.12 & 601.48 \\
\hline \multirow[t]{2}{*}{2} & $\begin{array}{c}\text { Removing the first } \\
3 \text { terms }\end{array}$ & 80.22 & 373.95 & 864.53 & 3361.53 & 79.10 & 366.13 & 1592.84 & 6085.75 & 36.63 & 187.00 \\
\hline & $\begin{array}{l}\text { Removing the first } \\
6 \text { terms }\end{array}$ & 13.71 & 80.50 & 29.20 & 174.95 & 13.91 & 85.79 & 44.84 & 274.04 & 13.56 & 73.85 \\
\hline
\end{tabular}

Number 1 represents the planar window glass, and number 2 represents the optical planar element.

\section{Discussion and Conclusions}

We compared three methods in this study. Inaccurate system calibration is one of the major error sources in PMD, which limits its practical applications. In EPCM, the camera position is no longer calibrated by Zhang's technology; the entrance pupil center is accurately calculated as a point source by EPCM. The measurement accuracy is high for measuring the low order terms of the surface if the experimental misalignment can be controlled very well. In PM, the accuracy of Zhang's technique is easily affected by the quality of the calibration target and the accuracy of feature point extraction. There are also too many parameters to be addressed in the optimization of the non-linear camera model, which makes it difficult to accurately obtain the projection center of the camera lens. The projection center obtained by this method is unstable, but the accuracy is high after eliminating the systematic error [10]. In MPMD, the pre-calibration result is only used as an initial value in the post-optimization, and the screen pose can be optimized with the shape estimation for a mono-PMD system. If a good pre-calibration is obtained, the MPMD method will obtain a relatively low shape error. However, the reconstruction result will be worse if the pre-calibration is performed poorly, including an initial guess far from the reality. 
It is generally believed that good results of camera calibration imply a higher accuracy of the reconstructed surface shape. Hence, some methods $[7,8,11,15,21]$ have been developed to calibrate parameters by reducing the calibration error as much as possible. This paper shows that EPCM has a higher measurement accuracy in comparison with the other two methods.

Author Contributions: Conceptualization, R.G. and D.L.; methodology, R.G. and D.L.; software, R.G., D.L., X.Z., R.W. and W.Z. (Wanxing Zheng); data curation, R.G., D.L. and X.Z.; writing-original draft preparation, R.G.; writing-review and editing, D.L., X.Z., R.W., X.L. and W.Z. (Wuxiang Zhao); All authors have read and agreed to the published version of the manuscript.

Funding: This research was funded by National Natural Science Foundation of China (grant number: U20A20215 and 61875142) and Sichuan University (grant number: 2020SCUNG205).

Institutional Review Board Statement: Not applicable.

Informed Consent Statement: Not applicable.

Data Availability Statement: Not applicable.

Conflicts of Interest: The authors declare no conflict of interest.

\section{Appendix A}

In Figure A1, the experimental setup for measuring the entrance pupil position is composed of a light source, the camera lens, and a microscope, which is used to measure the distance between the entrance pupil and the front surface of the camera lens. In the measurement, when the microscope is adjusted to focus on the entrance pupil and the first surface of the camera lens, respectively, two positions are recorded. Considering the distance between the external stop and the front surface of camera lens, the distance $\left(d_{p}\right)$ between the external stop and the entrance pupil of the camera lens can be obtained, as $d_{p}=23.96 \mathrm{~mm}$.

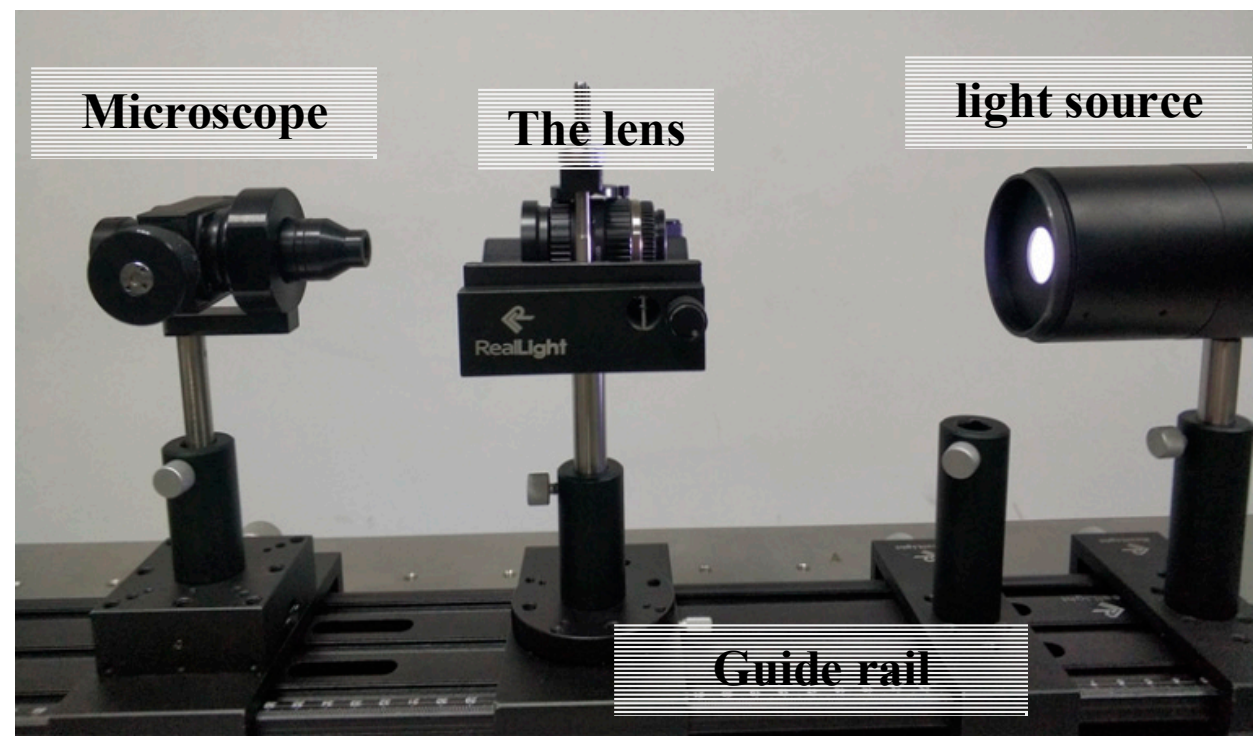

Figure A1. Experimental setup for measuring the distance $d_{p}$. 


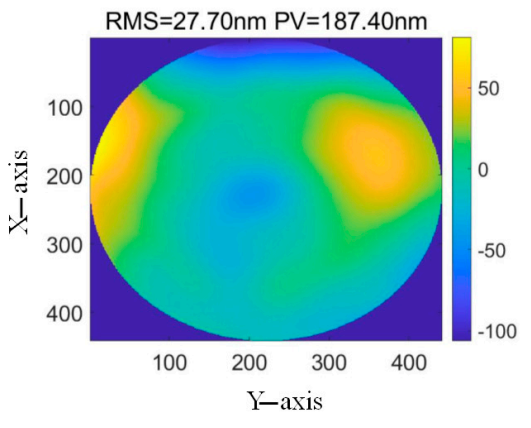

(a)

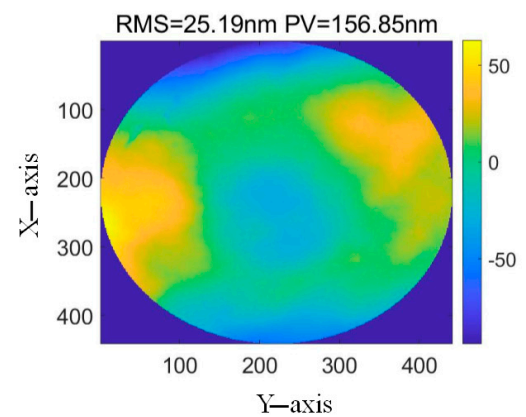

(b)

Figure A2. (a) Reconstruction results obtained by using our method. (b) Reconstruction results obtained by the interferometer.

\section{References}

1. Markus, M.C.; Knauer, J.; Hausler, G. Phase measuring deflectometry: A new approach to measure specular free-form surfaces. SPIE Photonics Eur. 2004, 5457, 366-376.

2. Huang, L.; Idir, M.; Zuo, C.; Asundi, A. Review of phase measuring deflectometry. Opt. Lasers Eng. 2018, 107, 247-257. [CrossRef]

3. Zhang, Z.; Wang, Y.; Huang, S.; Liu, Y.; Chang, C.; Gao, F.; Jiang, X. Three-dimensional shape measurements of specular objects using phase-measuring deflectometry. Sensors. 2017, 17, 2835. [CrossRef] [PubMed]

4. Su, P.; Parks, R.E.; Wang, L.R.; Angel, R.P.; Burge, J.H. Software configurable optical test system: A computerized reverse Hartmann test. Appl. Opt. 2010, 49, 4404-4412. [CrossRef]

5. Su, P.; Khreishi, M.; Su, T.Q. Aspheric and freeform surfaces metrology with software configurable optical test system: A computerized reverse Hartmann test. Opt. Eng. 2014, 53, 031305.

6. Wang, R.Y.; Li, D.H.; Li, L.; Xu, K.Y.; Tang, L.; Chen, P.Y.; Wang, Q.H. Surface shape measurement of transparent planar elements with phase measuring deflectometry. Opt. Eng. 2018, 57, 104104. [CrossRef]

7. Huang, L.; Xue, J.P.; Gao, B. Modal phase measuring deflectometry. Opt. Express 2016, 24, 24649-24664.

8. Xu, Y.; Gao, F.; Jiang, X. A brief review of the technological advancements of phase measuring deflectometry. PhotoniX 2020, 1, 1-10. [CrossRef]

9. Chen, P.Y.; Li, D.H.; Wang, Q.H.; Li, L.; Xu, K.Y.; Zhao, J.G.; Wang, R.Y. A method of sub-aperture slope stitching for testing flat element based on phase measuring deflectometry. Opt. Laser. Eng. 2018, 110, 394-400. [CrossRef]

10. Kewei, E.; Li, D.H.; Yang, L.J.; Guo, G.R.; Li, M.Y.; Wang, X.M.; Zhang, T.; Xiong, Z. Novel method for high accuracy figure measurement of optical flat. Opt. Laser. Eng. 2017, 88, 162-166.

11. Huang, R.; Su, P.; Burge, J.H.; Idir, M. X-ray mirror metrology using SCOTS/deflectometry. Proc. SPIE 2013, 8848, 88480G.

12. Huang, R.; Su, P.; Burge, J.H.; Huang, L.; Idir, M. High-accuracy aspheric X-ray mirror metrology using Software Configurable Optical Test System/deflectometry. Opt. Eng. 2015, 54, 84103. [CrossRef]

13. Luhmann, T. Close range photogrammetry for industrial applications. ISPRS J. Photogramm. Remote Sens. 2010, 65, 558-569.

14. Sun, T.; Xing, F.; You, Z. Optical system error analysis and calibration method of high-accuracy star trackers. Sensors 2013, 13, 4598-4623. [CrossRef]

15. Xiao, Y.; Su, X.; Chen, W. Flexible geometrical calibration for fringe-reflection 3D measurement. Opt. Lett. 2012, 37, 620-622. [CrossRef] [PubMed]

16. Zhang, Z. A Flexible New Technique for Camera Calibration. IEEE Trans. Pattern Anal. Mach. Intell. 2000, $22,1330-1334$. [CrossRef]

17. Hou, Y.; Su, X.; Chen, W. Alignment Method of an Axis Based on Camera Calibration in a Rotating Optical Measurement System. Appl. Sci. 2020, 10, 6962. [CrossRef]

18. Sasián, J. Introduction to Aberrations in Optical Imaging Systems; Cambridge University Press: Cambridge, UK, 2011.

19. Gennery, D.B. Generalized Camera Calibration Including Fish-Eye Lenses. Int. J. Comput. Vis. 2006, 68, 239-266. [CrossRef]

20. Kumar, A.; Ahuja, N. Generalized Pupil-centric Imaging and Analytical Calibration for a Non-frontal Camera. Comput. Vis. Pattern Recognit. IEEE 2014, 3970-3977.

21. Kumar, A.; Ahuja, N. On the Equivalence of Moving Entrance Pupil and Radial Distortion for Camera Calibration. In Proceedings of the International Conference on Computer Vision (ICCV), Santiago, Chile, 7-13 December 2015.

22. Li, D.H.; E, K.W.; Li, M.Y. Study on the surface shape measurement of planar element based on slope sensor method. Acta Optica Sin. 2014, 34, 192-199.

23. Zuo, C.; Feng, S.; Huang, L. Phase shifting algorithms for fringe projection profilometry: A review. Opt. Lasers Eng. 2018, 109, 23-59. [CrossRef] 
24. Wang, Z.; Han, B. Advanced iterative algorithm for phase extraction of randomly phase-shifted interferograms. Opt. Lett. 2004, 29, 1671-1673. [CrossRef] [PubMed]

25. Zhao, C.; Burge, J.H. Orthonormal vector polynomials in a unit circle, part I: Basis set derived from gradients of Zernike polynomials. Opt. Express 2007, 15, 18014-18024.

26. Zhao, C.; Burge, J.H. Orthonormal vector polynomials in a unit circle, part II: Completing the basis set. Opt. Express 2008, 16, 6586-6591. [CrossRef]

27. Noll, R.J. Zernike polynomials and atmospheric turbulence. J. Opt. Soc. Am. 1976, 66, 207-211.

28. Wang, R.Y.; Li, D.H.; Zhang, X.W. Systematic error control for deflectometry with iterative reconstruction. Measurement 2020, 168, 108393. [CrossRef]

29. Bouguet, J.Y. Camera Calibration Toolbox for Matlab. Available online: http://www.vision.caltech.edu/bouguetj/calib_doc/S (accessed on 14 October 2015).

30. Korsch, D.; Hunter, W.R. Reflective Optics; Academic Press: Cambridge, MA, USA, 1991. 